\title{
Multiscale Additive Manufacturing of Metal Microstructures
}

\section{Journal Article}

Author(s):

Ercolano, Giorgio; Zambelli, Tomaso; van Nisselroy, Cathelijn (D); Momotenko, Dmitry; Vörös, Janos (D); Merle, Thibaut; Koelmans, Wabe W.

Publication date:

2020-02

\section{Permanent link:}

https://doi.org/10.3929/ethz-b-000383568

\section{Rights / license:}

In Copyright - Non-Commercial Use Permitted

\section{Originally published in:}

Advanced Engineering Materials 22(2), https://doi.org/10.1002/adem.201900961 
This is the pre-peer reviewed version of the following article: G. Ercolano, T. Zambelli, C. van

Nisselroy, D. Momotenko, J. Vörös, T. Merle, W. W. Koelmans; "Multiscale Additive

Manufacturing of Metal Microstructures", Adv. Eng. Mater., 2019, 1900961, which has been published in final form at https://doi.org/10.1002/adem.201900961. This article may be used for non-commercial purposes in accordance with Wiley Terms and Conditions for Use of SelfArchived Versions.

\section{Multiscale Additive Manufacturing of Metal Microstructures}

WILEY-VCH

Giorgio Ercolano*, Tomaso Zambelli*, Cathelijn van Nisselroy, János Vörös, Thibaut Merle, Wabe W. Koelmans

Dr. Giorgio Ercolano, Thibaut Merle, Dr. Wabe W. Koelmans

Exaddon AG, Sägereistrasse 25, 8152, Glattbrugg, Switzerland

E-mail: giorgio.ercolano@exaddon.com

Prof. Tomaso Zambelli, Cathelijn van Nisselroy, Prof. János Vörös, Laboratory of Biosensors and Bioelectronics, ETH Zürich, Gloriastrasse 35, 8092, Zurich, Switzerland

E-mail: ztomaso@ethz.ch

Keywords. additive manufacturing, electrochemical microprinting, micro 3D printing, metal, microcoils, FluidFM

Abstract. We show that the electrochemical metal printing system based on hollow AFM cantilevers for the local delivery of precursor species is capable to cover additive manufacturing at different length scales, from sub-micrometer to sub-millimeter, within the same printed object by dynamically adjusting printing parameters while keeping the same nozzle diameter. We rationalized the interplay between the lateral voxel dimensions, nozzle aperture and pressure, while keeping other deposition parameters fixed. We demonstrate an accurate control of the voxel area over two orders of magnitude during printing with the same nozzle. Such control was enabled by integration of on-the-fly adaptation of the voxel size by an order of magnitude, which is steered by the applied pressure. Capabilities of this printing technique are highlighted by fabrication of a helix of four copper wires in a layer-by-layer fashion whereby each wire is printed with a different diameter. These results pave the way for a significant throughput increase by the careful adjustment of voxel dimensions for different features within the same object, allowing higher fabrication speed for larger structures, whilst keeping high enough spatial resolution for its delicate parts. 


\section{WILEY-VCH}

\section{Introduction}

Additive manufacturing (AM) of metal objects at the micrometer scale is a rapidly growing research field with potential applications in optics, micro-robotics and microelectronics [1-5]. Techniques relying on local electrochemical reduction of metallic ions are considered among the most advantageous since they offer the benefits of a single-step fabrication without pre- or post-processing (such as sintering), and produce fully dense, conductive and mostly contaminant-free metal features. Electrochemical (ec) 3D printing methods are based on confinement of the electrochemical reaction in a small volume by either using a droplet cell configuration, where the printing is localized within a liquid meniscus $[5-8][2,3,4]$, or by a local delivery of precursor species in liquid $[9,10]$. The former approach requires a glass pipette filled with the metal ion solution and equipped with a counter electrode. A meniscus, which is formed between the pipette aperture and the substrate when the pipette is positioned in its proximity, confines electroplating into a droplet footprint. The latter approach is based on local dispensing of metal ions in liquid, which can be achieved with a either a double-barrel pipette or with a FluidFM probe [11]. In the first case, both barrels have a separate counter electrode, whereas one barrel is filled with the metal ion solution and the second barrel with an inert electrolyte. This inert solution regulates the ionic current to be fed into the feedback loop that controls the distance between the pipette and the working electrode. In the second case, a microchanneled atomic force microscope (AFM) cantilever, also known as a FluidFM probe, containing a metal ion solution, is approached to the working electrode of a standard threeelectrode cell using the cantilever deflection as feedback signal. This signal allows detecting the plating completions as the voxel that reaches completions underneath the aperture, pushes against the probe apex causing it to deflect the AFM cantilever. In this way, the printing method is fully automated achieving a true layer-by-layer process.

Although a few experiments were carried out with platinum [5,12], copper was so far the most popular choice for electroplating because of its high coulombic efficiency (minimum 


\section{WILEY-VCH}

faradaic losses due to side reactions) in a relatively wide voltage range [9]. As recent developments of the ec printing to be highlighted, the meniscus-confined protocol has been parallelized taking advantage of nozzle arrays to enlarge the printing area combined with a feedback control associated to the ionic current when the meniscus is formed $[7,13]$. Despite these efforts in ec printing a high volumetric throughput remains to be an essential and unaddressed requirement for industrial applications, in particular for electronics (circuits) and mechanical systems. Local electroreduction protocols all share a growth-speed limitation as dictated by the maximum plating rates. By increasing the later voxel dimensions (voxel area) the volumetric throughput increases, however, at the expense of spatial resolution, voiding the technology's main benefit. Assuming a constant (and optimized) voxel printing rate per nozzle, one recognizes that the ability to steer the voxel size on-the-fly increases fabrication rates and significantly advances the throughput. This steering enables printing larger features, within a structure design, at a higher volumetric rate making use of an increased voxel area, whilst keeping printing resolution high for fabrication of smaller features within the same structure design.

In this work, we demonstrate how the interplay of nozzle aperture and applied pressure enables adjusting the voxel size, bridging the sub-micron feature dimensions and submillimeter printing scales and accordingly increasing printing throughput.

\section{Results and Discussion}

\subsection{Design of the novel additive micromanufacturing system}

In this study we designed a novel microprinting setup. Our previous instrument was controlled with a custom LabVIEW interface [9] that synchronized all components of the system via data acquisition cards. This enabled complete automation and coordination of the probe positioning system (the $x-y-z$ piezopositioners of the AFM) with the liquid flow (set with the pressure controller), applied substrate potentials with a potentiostat, and the AFM deflection signal, 


\section{WILEY-VCH}

which is used to detect the voxel growth. However, the instrument suffered certain limitations, including the restriction in height of the printed features given by the maximum $\mathrm{z}$ piezo range $(15 \mu \mathrm{m})$, as well as some inherent latency in synchronization of instrumentation, leading to a relatively slow response (tens of milliseconds) resulting in overall slower printing rates.

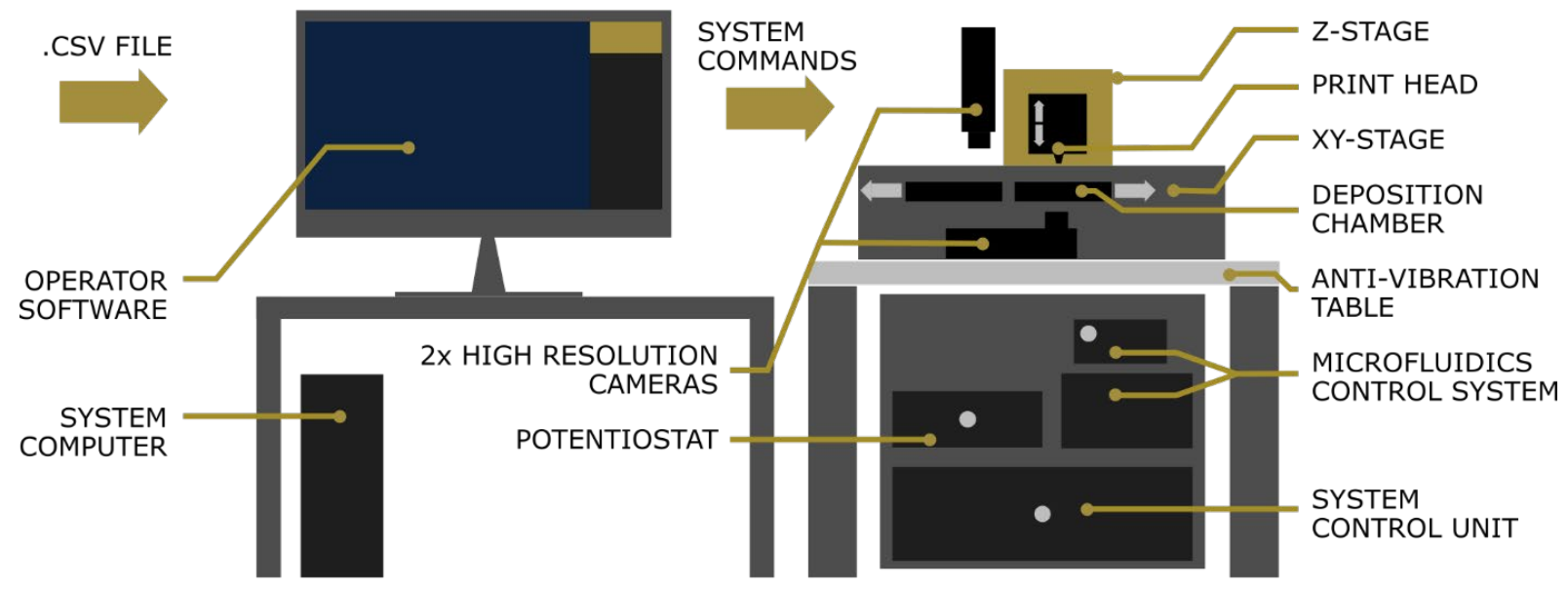

Figure 1. Schematic of the additive micromanufacturing system. On the left a system computer sends commands to the system control unit on the right. On the right, the system control unit governs the printing process using an embedded controller. The FluidFM probe sits on the printing head and is moved into the deposition chamber by the z-stage. A microfluidics control system regulates the flow of electrolyte through the aperture of the cantilever.

Here, we introduce a completely re-designed system from both the hardware and the software perspective (Figure 1). A dedicated embedded controller (Exaddon AG, CH) is specifically designed and programmed for the additive micromanufacturing process. A first feature is the very low latency and fast feedback loop: when the deflection threshold is exceeded due to the voxel touching the tip apex, the controller responds within $1 \mathrm{~ms}$ by issuing a command to retract the tip from the voxel surface and move to the next point. Such speed is of strategic importance because it increases the probe lifetime (by minimizing clogging of the aperture) and allows for printing at higher rates. A second feature is the stable and long-term operation: as 3D volume bodies may take some time to complete, the new system is conceived to print continuously for prolonged periods of time, up to tens of hours. To that end, the embedded controller has a buffer containing the next 16 voxel coordinates. The system is therefore to a large degree resilient towards any communication delay caused by the computer 


\section{WILEY-VCH}

operating system that may execute many other tasks at any moment in time. Further parts of the hardware of the new system are two high-resolution optical systems (top and bottom view) for loading of the nozzle, for printer adjustment and calibration, as well as for in situ visualization of the printed structures. Live video is streamed to the system software for real-time operation. Computer-assisted alignment is built-in for printing on already existing structures. The latter allows the user to print on, for example, integrated electrodes that are pre-defined on a chip surface. The maximum volume of the printing space is $200 \mathrm{~mm} \times 70 \mathrm{~mm} \times 60 \mathrm{~mm}$ assured by a three axes positioning stage (Exaddon $\mathrm{AG}, \mathrm{CH}$ ) constituted of linear motors with an $x-y$ positioning accuracy (full-range, repeatability) of $\pm 250 \mathrm{~nm}$ as well as a $z$ motor with $5 \mathrm{~nm}$ positioning accuracy at a $0.1 \mathrm{~nm}$ sensor resolution achieving a positioning time from a voxel to the following one in under $50 \mathrm{~ms}$ (only $z$ motion). The printing chamber (i.e. the three-electrode cell, see Supporting Information Figure S1) was redesigned in a modular fashion with the parts made out of solid Teflon, a working electrode that is connected to the printing substrate of $12 \mathrm{~mm} \times 12 \mathrm{~mm}$, a graphite counter electrode, and a $\mathrm{Ag} / \mathrm{AgCl}$ wire used as reference. The instrument is operated with a dedicated software that controls the printer, prints designs and stores the log data (see Supporting Information Figure S2).

\subsection{Voxel size and volumetric deposition speed as function of the overpressure for different apertures}

Our additive micromanufacturing system is a multiparameter instrument, whereby the nozzle aperture, the applied pressure $p$, the electrodeposition potential $E$ as well as the voxel height $\Delta z$ can be finely tuned. We focused on a printing speed in the $z$ direction of the order of $1 \mu \mathrm{m} \mathrm{s}^{-1}$ with $E$ and $\Delta z$ kept constant while varying nozzle opening size and pressure $p$. 


\section{WILEY-VCH}

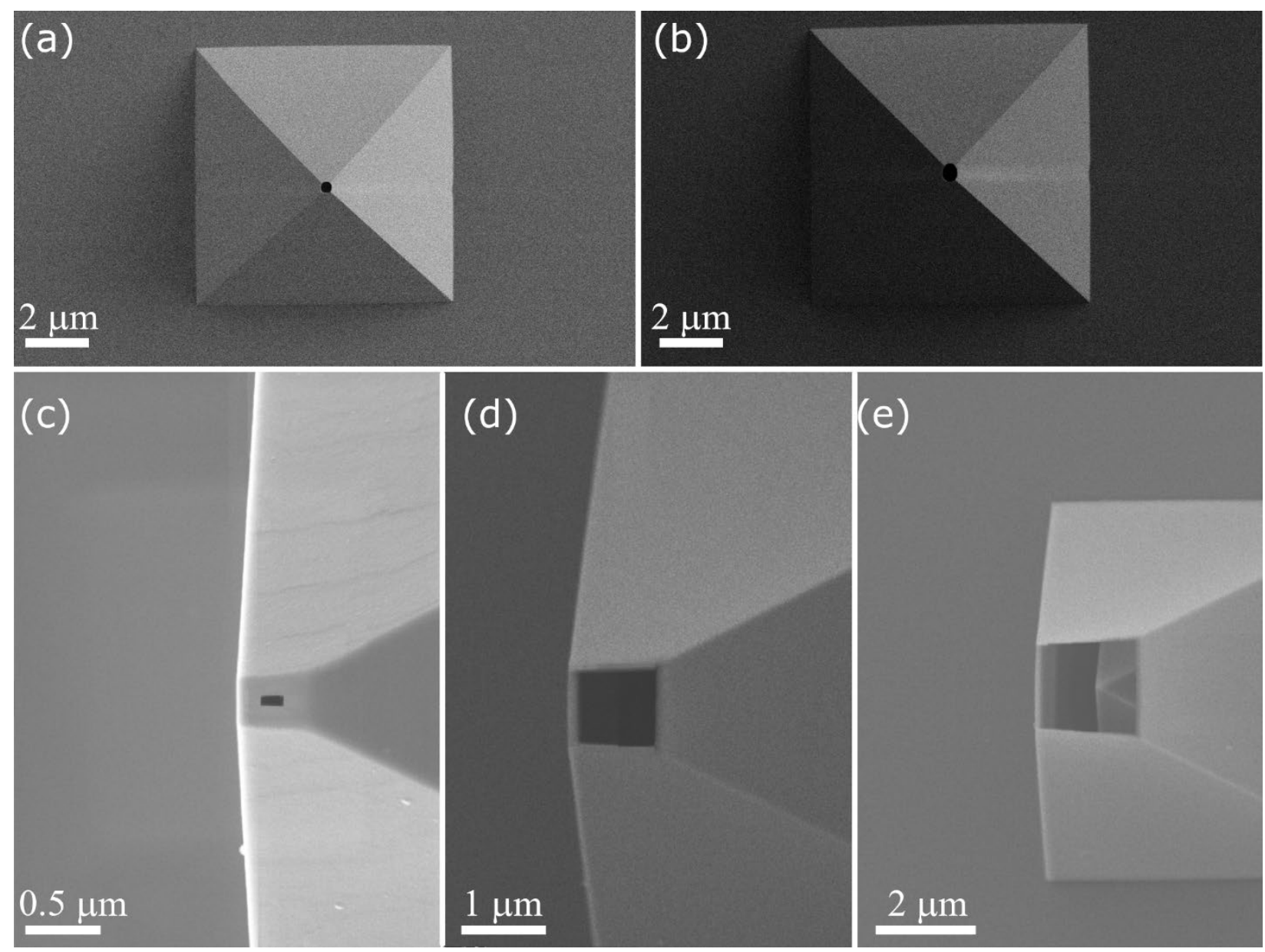

Figure 2. SEM images of the different tip aperture. Top views of a (a) $300 \mathrm{~nm}$ and a (b) 500 $\mathrm{nm}$ diameter aperture obtained by contact lithography, (c) $100 \mathrm{~nm}$ (d) 1 um and (e) 2 um side square apertures obtained by FIB milling of closed piramidal probes.

We had at our disposal pyramidal hollow probes with 300 and $500 \mathrm{~nm}$ circular apertures (Figure 2a,b) lithographically obtained on a wafer scale as well as aperture-less pyramidal hollow probes $[14,15]$. In the latter case, the apertures were milled by focused ion beam (FIB) to form square-shaped openings with a side of $100 \mathrm{~nm}, 1 \mu \mathrm{m}$, and $2 \mu \mathrm{m}$ (Figure 2c-e, see Experimental Section). The choice of the smallest aperture dimension of $100 \mathrm{~nm}$ was conditioned to avoid potential complications in filling the probes with electrolyte [16], whereas $2 \mu \mathrm{m}$ was the maximum size possible due to the limit set by the presence of the apex of the inner pyramid (discernable in Figure 2e).

These cantilever probes were employed to electrodeposit copper features. In general, copper could be printed in the window from $-400 \mathrm{mV}$ to at least $-900 \mathrm{mV}$ (potentials here are reported with respect to $\mathrm{Ag} / \mathrm{AgCl}$ wire). At $-500 \mathrm{mV}$ the plating speed $\dot{z}$ is $\sim 1 \mu \mathrm{m} \mathrm{s}^{-1}$ and structures could 


\section{WILEY-VCH}

be printed it the pressure range $10-500$ mbar, whereas at much more negative voltages, and thus much higher plating speeds, the pressure range that can be addressed is compromised due to the increased clogging risk by metal growth inside the aperture.
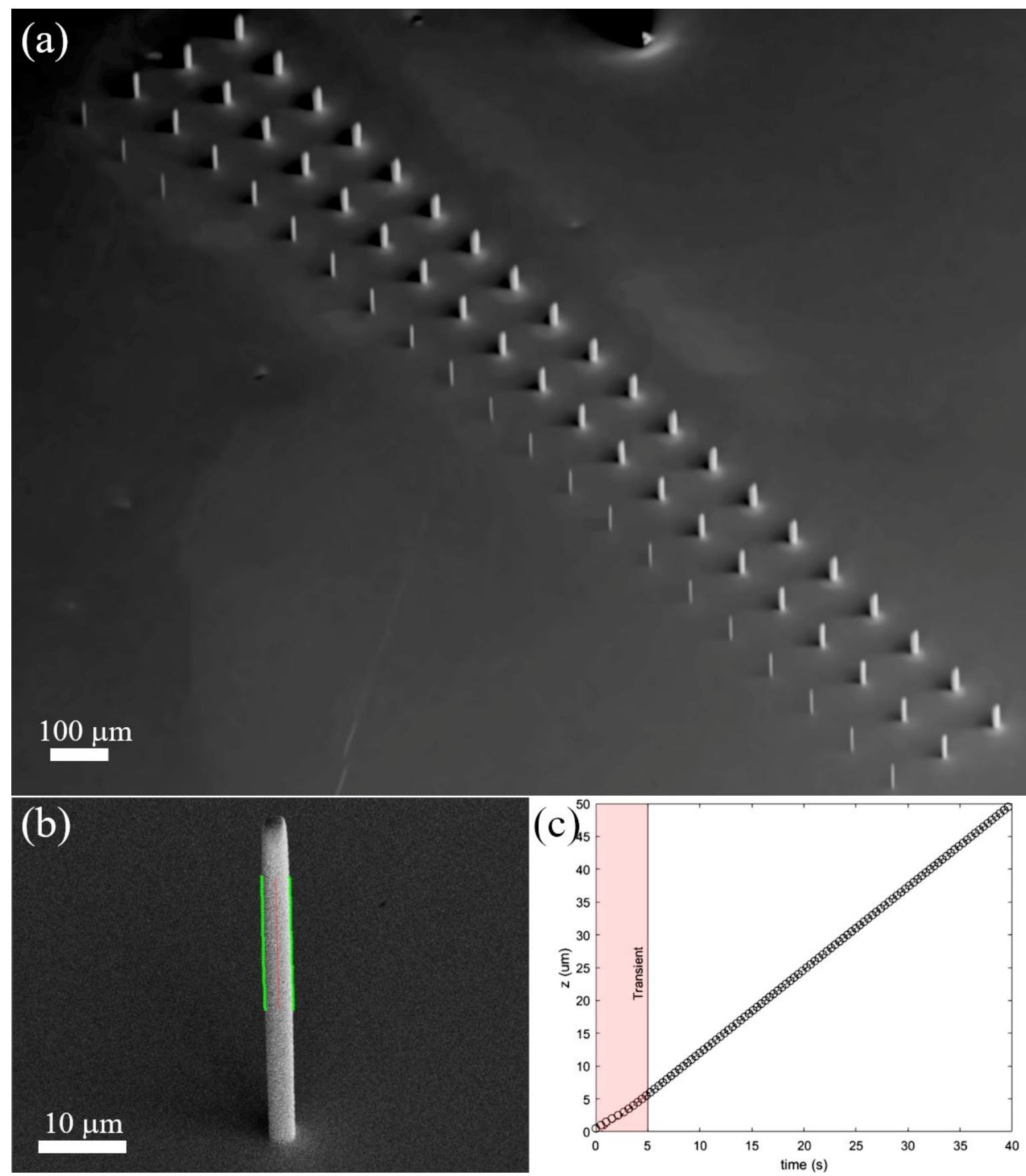

Figure 3. (a) SEM image of a field of pillars printed at decrasing pressure values, (b) SEM image a single pillar with overlaid the results of the automated diameter analysis, and (c) height over time during the print of the pillar fitted to a linear function (Vertical plating speed determination).

We initially assessed the printing capabilities of the new printing instrument by depositing $\mathrm{Cu}$ in a form of pillars. The pillar array shown in Figure 3a was plated in a voxel-by-voxel 


\section{WILEY-VCH}

manner: after detecting a successful growth of a voxel, the tip was each time retracted by a $\Delta z$ of $500 \mathrm{~nm}$. This retraction distance is small enough to achieve sufficient confinement of the localized delivery of metal ions, which eliminates broadening of the printed features, yet large enough to allow for voxel printing times of $\sim 500 \mathrm{~ms}$, about an order of magnitude longer than the printer repositioning $(\sim 50 \mathrm{~ms})$. We kept the $\Delta \mathrm{z}$ of $500 \mathrm{~nm}$ constant throughout all the experiments, leading to print rates of about 2 voxels $\mathrm{s}^{-1}$.

For each aperture size, $50-\mu \mathrm{m}$-tall pillars (100 voxels) were printed at different pressures, in a range from 500 to 10 mbar with a step size of 10 mbar. At pressures higher than 500 mbar, especially in combination with the larger probe apertures, the printing process was observed to become less controllable due to hydrodynamic effects interfering with the voxel detection. Pillars are generally cylindrical for all aperture shapes, however, a slight trace of the geometry of the square apertures is observed at very low pressures $(\mathrm{p}<30$ mbar). For each pillar, the diameter $d$ was estimated along the main axis (Figure 3b). Furthermore, using the data stored in the log file, generated by the system control software, the pillar height can be plotted as function of the elapsed time (Figure 3c). It can be noticed that the growth is not completely linear in the first $5 \mu \mathrm{m}$, revealing a transient onset, while further proceeding in a linear way. 


\section{WILEY-VCH}
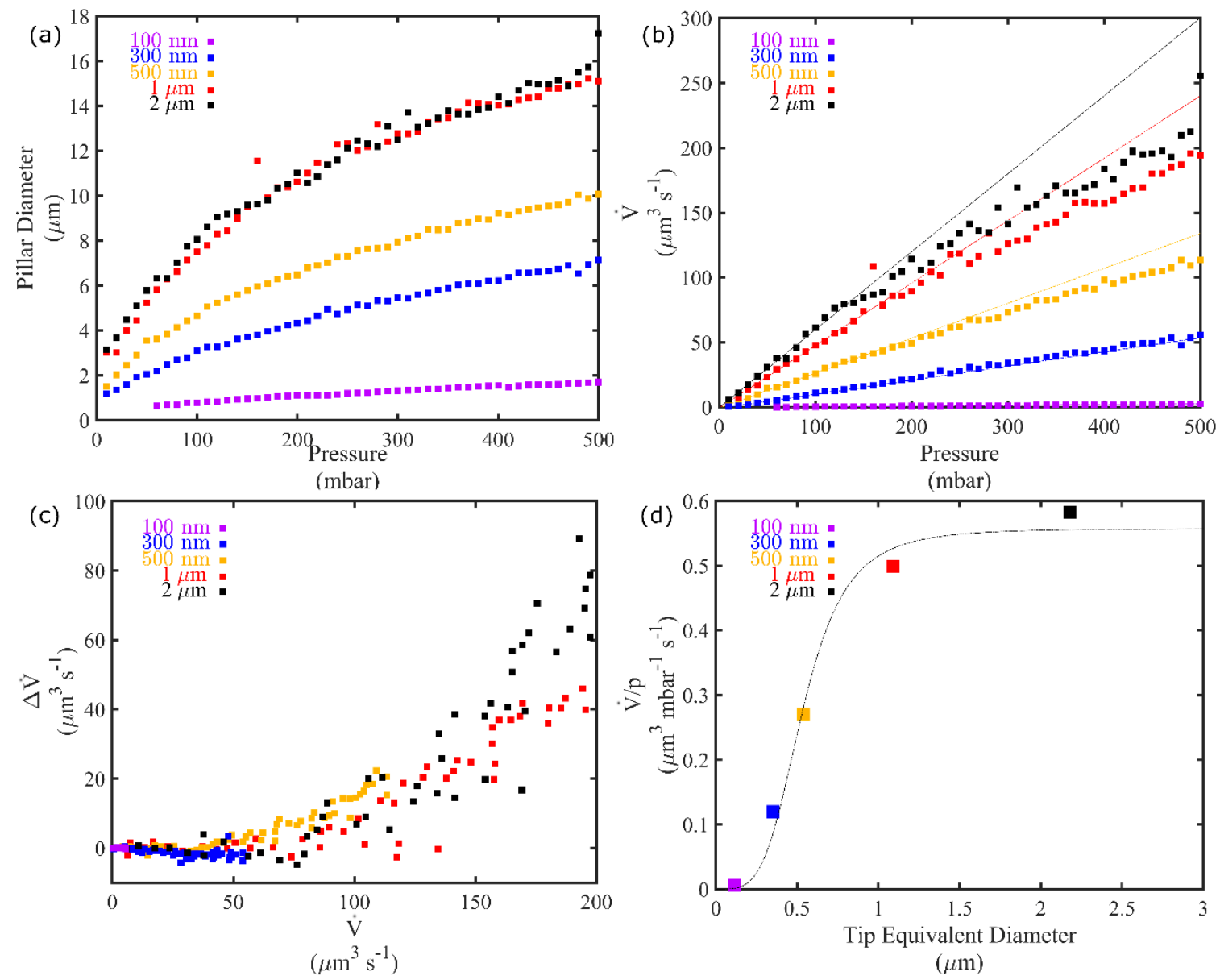

Figure 4. (a) The pillar diameters obtained as a function of the pressure applied during printing, (b) the volumetric deposition speed as function of the pressure, (c) the fitting error of a linear fit applied on the volumetric deposition speed of (b), plotted as a function of the volumetric deposition speed (d) the volumetric deposition speed over pressure for the different tip equivalent diameters.

We further analyzed these printing results in terms of the pillar diameter (Figure 4a), the plating speed as well as the volumetric deposition speed (in $\mu \mathrm{m}^{3} \mathrm{~s}^{-1}$, calculated as $\dot{V}=\frac{\pi}{4} d^{2} \dot{z}$, Figure 4b), as a function of the applied pressure $p$. At low pressures, $p<100$ mbar, the volumetric deposition speed $\dot{V}$ increases linearly, whereas at high pressures, $p>100$ mbar, $V$ begins to saturate (i.e., the derivative $\mathrm{d} \dot{V} / \mathrm{d} p$ gradually decreases). From Figure 4a, it can be inferred that the pillar diameter for each aperture can be tuned in the range of almost one order of magnitude by adjusting the pressure between 10 and 500 mbar (the diameter changes from $\sim 1 \mu \mathrm{m}$ at 10 mbar to $\sim 10 \mu \mathrm{m}$ at $500 \mathrm{mbar}$ for the $500 \mathrm{~nm}$ nozzle). The pillar diameter is assumed to be strictly related to the voxel diameter. We recognize that, in general, the voxel shape and 


\section{WILEY-VCH}

size are a function of its underlying geometry. However, the simple case of a pillar offers a useful benchmark, i.e. the pillar diameter becomes an accurate measure of the voxel diameter.

In a more general view, operating the same tool and varying two parameters (the aperture size and the applied pressure), in a range where the control of the printing process is unaltered, a voxel diameter is obtained spanning in a range of almost two orders of magnitude. Such a range is equivalent to a voxel area range, and therefore the range in throughput $\left(\mu \mathrm{m}^{3}\right.$ of $\left.\mathrm{Cu} \mathrm{s}^{-1}\right)$ of over three orders of magnitude. Compared to meniscus-confined plating $[7,13]$ we note that scaling with aperture size is there also demonstrated over three orders of magnitude, however, controlled scaling of the voxel area using the same nozzle is not demonstrated. It is evident that changing a nozzle is slow and hampers throughput, much more than changing a process parameter like the pressure, which can be done on-the-fly.

Inspecting Figure 4b, we notice that $\dot{V}(p)$ deviates from a linear relation at higher pressures, indicating that at higher pressures a smaller fraction of the ejected $\mathrm{Cu}$ ions is electroplated on the pillars.

Next, the flow through the microchannel was theoretically assessed by means of an analytical model for the laminar flow of Newtonian fluids through a rectangular (100 nm, $1 \mu \mathrm{m}$ and $2 \mu \mathrm{m}$ fibbed tip) and an elliptical duct (300 $\mathrm{nm}$ and $500 \mathrm{~nm}$ tip), Hagen-Poiseuille equation). The simplest way to evaluate the flow through the cantilever is to model the microchannel and the tip opening as two separate ducts in series, each of them with its own flow resistance. The hydraulic resistance of the microchannel does not change with the tip opening. The tip opening's resistance is proportional to the $4^{\text {th }}$ power of the aperture equivalent diameter. Additionally, considering the plating efficiency as constant over the experimental series we can assume that the flow is proportional to the $\dot{V}$ measured. In this way the $\dot{V}$ - $p$ ratio can be directly related to the tip aperture equivalent diameter $D_{\text {e. }}$ (Supplemental Information, Equations 19). 


\section{WILEY-VCH}

It was confirmed that at low $p$ (or more precisely at low $\dot{V}$ ) the relation between $\dot{V}$ and $p$ is indeed linear, indicating that there is no change in the plating efficiency. On the contrary, at high $\dot{V}$ a loss of linearity is observed indicating a reduction in plating efficiency.

The plating speed $\dot{z}$ and consequently the volumetric deposition speed $\dot{V}$ are directly derived from the experimental data of Figure 3c and Figure 4a.

In Figure 4b, the volumetric deposition speed $\dot{V}=\dot{z} A$ with $A=\frac{\pi}{4} d^{2}$ is displayed as a function of pressure. The straight lines are the linear fits for $p<100$ mbar corresponding to the case where the overall plating efficiency is constant with the flow and therefore with the pressure. It can be noted that the smaller the nozzle aperture the wider the range of $p$ in which the plating efficiency is invariant with the pressure. It appears that the deviation from linearity is strictly linked to the volumetric deposition speed and the extended linear range of the $100 \mathrm{~nm}$ aperture tip is a direct consequence of smaller deposition rate $\dot{V}$ values accessible with such small apertures. Indeed, in Figure 4c the deviation from linearity is plotted against $\dot{V}$ for all the apertures and it is evident that no matter which tip is used, this deviation is only a function of $\dot{V}$ and therefore of the flow. For the $100 \mathrm{~nm}$ aperture, $\dot{V}$ is so small with respect to the values for the other apertures that it appears flattened on the $\mathrm{x}$ axis, a corresponding zoom in is proposed in the Figure 3S of the Supplemental Information, where its linear behavior can be discerned in the range from 50 to 500 mbar.

Figure $4 \mathbf{c}$ indicates a reduction in plating efficiency with the volumetric deposition speed, because at higher flows a decreasing fraction of the supplied $\mathrm{Cu}$ ions is reduced. This decrease can be intuitively explained by considering that by the flow, ejected by a small aperture, is more spatially focused [17].

Finally, the ratio $\dot{V} / p$ (calculated in the linear range) is plotted as a function of the equivalent diameter of the tip aperture (Figure 4d). According to the simple fluid-dynamics model, presented in the Supporting Information, the data points were fitted with the curve 


\section{WILEY-VCH}

$$
\frac{\dot{V}}{p}=\frac{1}{C_{1}+\frac{C_{2}}{D_{\mathrm{e}}{ }^{4}}}
$$

where $C_{1}$ and $C_{2}$ are fitting coefficients and, $D_{\mathrm{e}}$ is the tip equivalent diameter. $C_{1}$ is proportional the fix hydraulic resistance of the cantilever channel while $C_{2}$ is related to hydraulic resistance of the aperture. This simple relation can be used to predict the voxel diameter as a function of pressure and tip equivalent diameter. The relation shows three different regimes. At very small apertures the flow is dominated by the aperture, at large apertures the flow is dominated by the microchannel and a transition region is found inbetween.

\subsection{Layer-by-layer printing at different apertures and pressures}

After the thorough analysis of the electrochemical printing process on simple pillars, we moved on to the corresponding fabrication of 3D microobjects: a structure was designed combining four intertwined helices with a pillar in the middle having on top a pyramid (Figure 5). 


\section{WILEY-VCH}

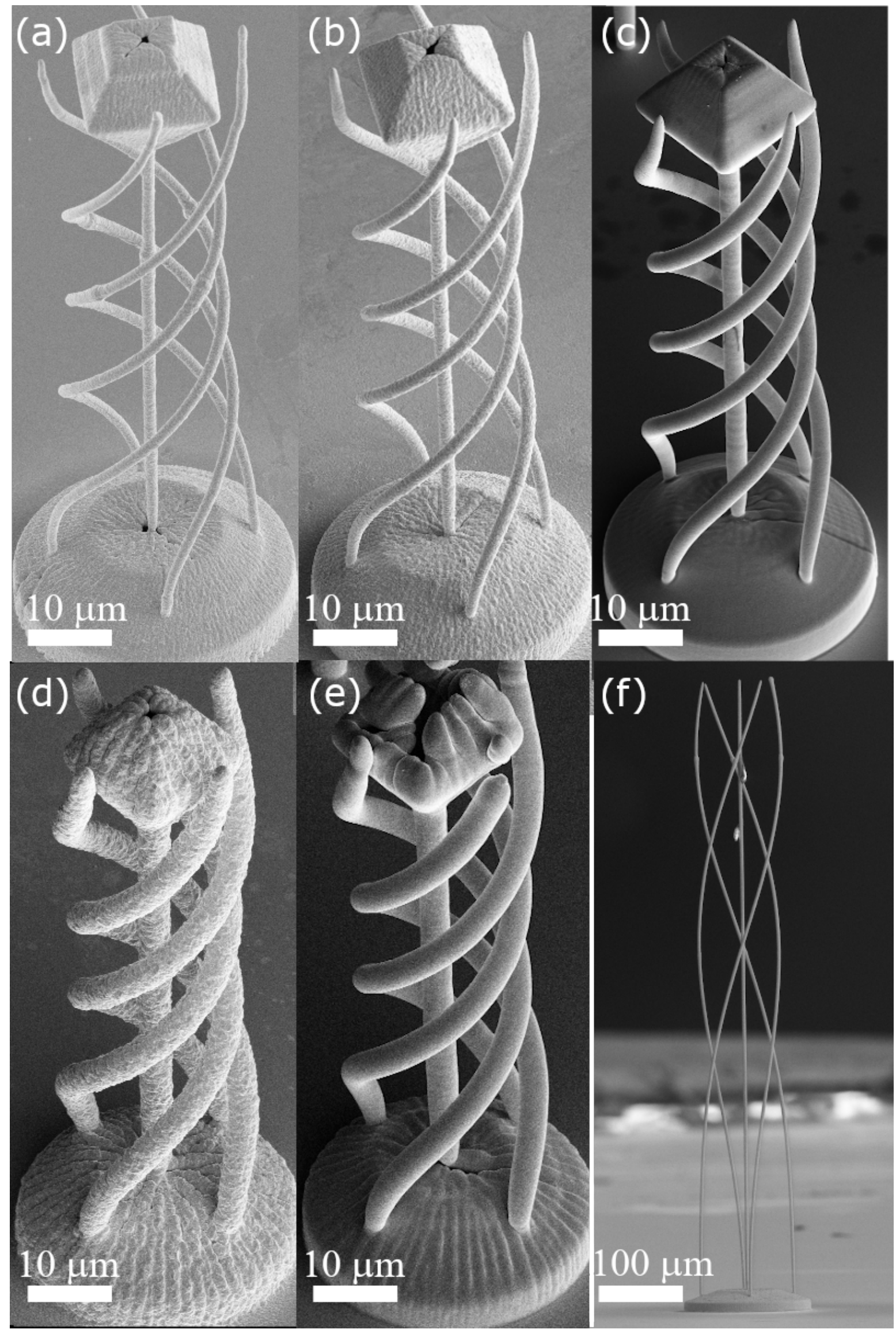

Figure 5. A demonstration structure printed with different tip apertures ranging in size from (a to e) $100 \mathrm{~nm}$ to $2 \mu \mathrm{m}$, (f) a larger $650 \mu \mathrm{m}$ tall structure was printed with a $300 \mathrm{~nm}$ tip, revealing an aspect ratio of 100:1 (strands). 


\section{WILEY-VCH}

The combination of the helices together with the pillar is thought to emphasize the true layerby-layer nature of our printing process, while the double truncated pyramid on top highlights the ability of the process to produce overhanging details too and, together with the base, is a demonstration of the ability to seamlessly transition from a 2D print mode (where a strand of voxels is merged together to form a smooth surface) to true $3 \mathrm{D}$ printing. The total number of voxels amounts to 9171. Aiming for fixed geometry and a fixed number of voxels, it is clear from Figure 4a, that a compromise had to be found considering which number of voxels and which size of the structure were chosen. We chose tovary the pressures to compensate as much as possible for the huge differences in nozzle area $\left(\sim 0.01 \mu \mathrm{m}^{2}\right.$ for a $100 \mathrm{~nm}$ nozzle and $\sim 4 \mathrm{~m}^{2}$ for a $2 \mu \mathrm{m}$ nozzle). In this example, we varied printing pressures values of 900 and 40 mbar for the 100 and $300 \mathrm{~nm}$ nozzles, respectively (Figure 5a and 5b), while we used 25 mbar for the three larger apertures (Figure 5c-e) to print the same voxel coordinate list. In case of smaller apertures relatively large overpressure values were set to maintain the voxel size required by the structure design. The overall printing took from $100 \mathrm{~min}$ to $35 \mathrm{~min}$ (for the smallest, 100 $\mathrm{nm}$, and the largest, $2 \mu \mathrm{m}$, aperture, respectively). The voxel area is uniform within each structure and, in agreement with the curves in Figure 4a, the structures printed by the larger nozzles are characterized by a lower resolution.

In addition, a large, $650-\mu \mathrm{m}$-tall structure with a $120-\mu \mathrm{m}$-diameter base and 3 - $\mu \mathrm{m}$-diameter strands, was printed at $100 \mathrm{mbar}$ with a $300 \mathrm{~nm}$ nozzle in $120 \mathrm{~min}$ (20K voxels) as a demonstration of the extent of the dimensional range of the new 3D printing instrument (Figure 5f).

\subsection{Voxel-by-voxel printing with each voxel at a different pressure}

Finally, to highlight the possibility of tailoring the voxel area by adjusting $p$, we designed a structure of four helixes, whereby each wire has a different diameter. Such object can be manufactured with a single nozzle only in a layer-by-layer fashion, requiring that each of the 


\section{WILEY-VCH}

four voxels of each layer is plated at a different value of pressure. For this, a $500 \mathrm{~nm}$ nozzle was operated at 20, 40, 60, and 80 mbar for each wire. In the SEM image of Figure 6, the four resulting wires are colored for better visualization.

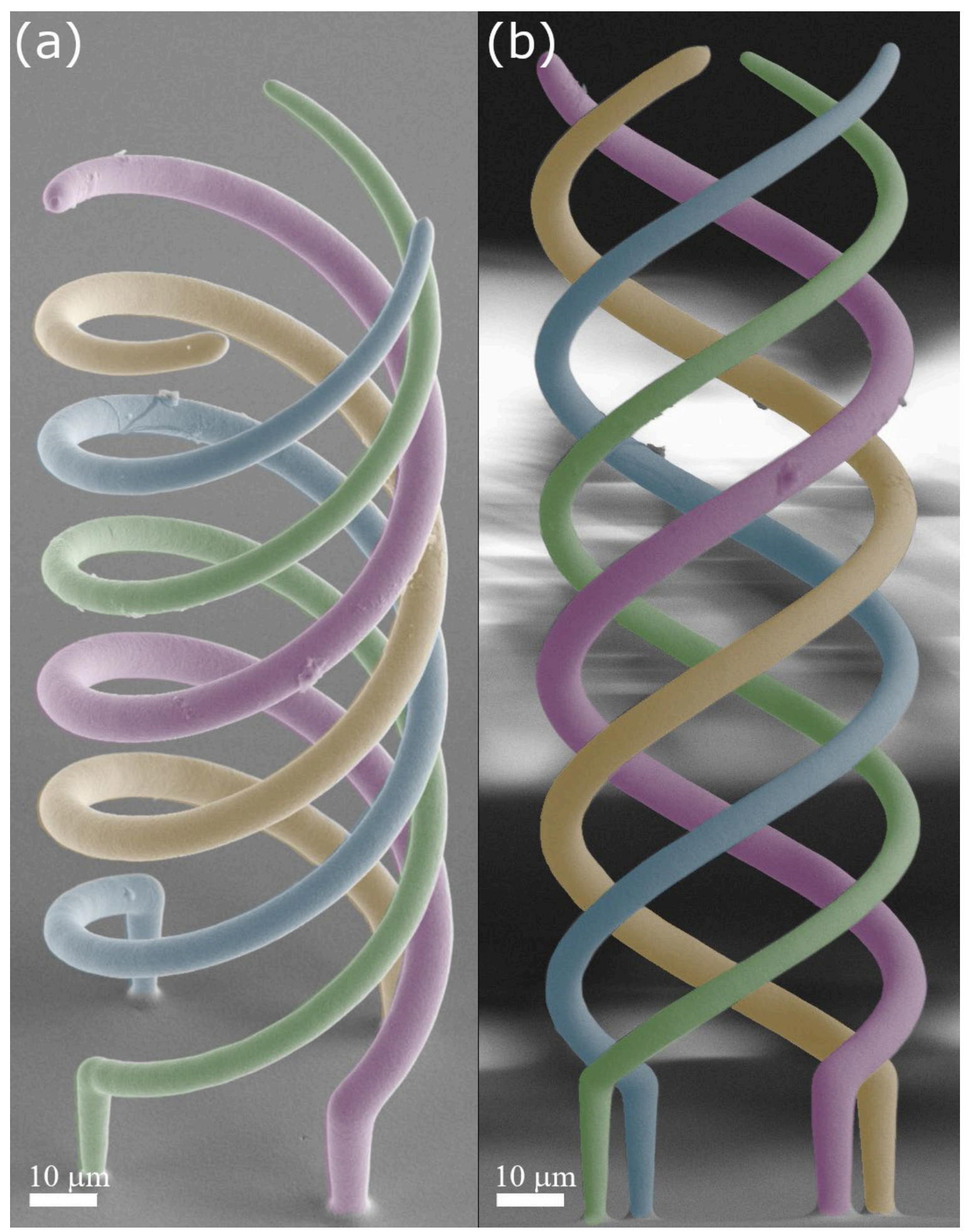

Figure 6. SEM images (colored) of four intertwined helices, each helix printed at a different pressure to showcase the flexibility and the ability to change and control the voxel diameter within the same structure. 3056 voxels were printed in 25 minutes to obtain the $180 \mu \mathrm{m}$ tall structures. (a) and (b) are two different views of the same object. 


\section{WILEY-VCH}

The unknown of this particular protocol is the time response of the pressure. Will the pressure and thus the cloud of $\mathrm{Cu}$ ions recover quickly enough when the nozzle is moved from a "violet" voxel at 80 mbar, the last of the four ones of each layer, back to a "green" voxel at 20 mbar, the first of each following layer? As evidenced from Figure 6, each wire has indeed a different diameter, which is homogeneous along all its length $(5,6,7$ and $8 \mu \mathrm{m}$, respectively). This difference undeniably attests that the positioning time is sufficiently large for the pressure to re-assume a smaller value.

\section{Conclusions}

A new additive micromanufacturing system was designed to optimize the electrochemical printing of copper by reducing the response and positioning times and increasing the motor range. While keeping fixed the electrochemical deposition potential and the voxel height $(\Delta z=$ $500 \mathrm{~nm}$ ), the influence of the applied pressure and the probe aperture on the lateral diameter of the electroplated copper voxels was investigated and rationalized. In this way, voxel area could be spanned over three orders of magnitude. More importantly, the voxel area could be spanned over two orders of magnitude using the same probe aperture. This multi-scale capability was exploited to fabricate copper microstructures $60 \times 60 \times 180 \mu \mathrm{m}^{3}$ and $120 \times 120 \times 650 \mu \mathrm{m}^{3}$ with a submicron resolution. Finally, a $50 \times 50 \times 170 \mu \mathrm{m}^{3}$ structure of four helicoidal wires was manufactured by changing the pressure on a voxel-by-voxel basis so that the four wires resulted each with a different diameter, but each homogeneous along the entire length. These results highlight a significant throughput increase with a possibility of tuning the voxel size depending on the feature dimensions within the same design. 


\section{WILEY-VCH}

\section{Experimental Section}

\section{Chemical solutions}

The supporting electrolyte was a $0.5 \mathrm{M} \mathrm{H}_{2} \mathrm{SO}_{4}$ with the addition of $\mathrm{HCl}$ to a concentration of $0.5 \mathrm{mM}$. The plating solution was a $0.8 \mathrm{M} \mathrm{CuSO}_{4}$ in $0.5 \mathrm{M} \mathrm{H}_{2} \mathrm{SO}_{4}$ solution. $\mathrm{CuSO}_{4} 5 \mathrm{H}_{2} \mathrm{O}$ copper (II) sulphate pentahydrate (Labkem), $\mathrm{H}_{2} \mathrm{SO}_{4}$ Sulfuric Acid 95-97\% (Labkem), $\mathrm{HCl}$ hydrochloric acid solution purum p.a. 24-26\% (Fluka) and, distilled water where used in the preparation of the supporting electrolyte and plating solution without further purification. The plating ink was filtered through a $0.2 \mu \mathrm{m}$ hydrophilic membrane filter (Millex).

\section{Working electrode}

The working electrode used were large with a 100 -nm-thick film of sputtered $\mathrm{Cu}$ on a $1.3 \times$ $1.3 \mathrm{~cm}^{2}$ silicon substrate with $13 \mathrm{~nm}$ of titanium adhesion layer. The copper films were protected from contamination and oxidation by a thin photoresist layer that was removed just prior the utilization.

\section{FluidFM probes and FIB}

The FluidFM-probes were mounted in a custom probe holder and coated with an $18 \mathrm{~nm}$ carbon layer using a CCU-010 Carbon Coater (Safematic) before milling by a FIB-SEM Nvision 40 device (Zeiss) with the SmartSEM software (Zeiss). The milled face of the pyramidal probe was aligned in parallel with the FIB-beam equipped with a gallium ion source. Subsequently, the probes were milled with an acceleration voltage of $30 \mathrm{kV}$ at milling currents varying from $10 \mathrm{pA}$ up to $80 \mathrm{pA}$, depending on the desired opening. The active milling process was followed in live SEM mode and was only terminated when the desired opening size was reached. After the milling process, the probes were glued onto a dedicated printing holder (Exaddon AG, Switzerland). 


\section{WILEY-VCH}

We have used three $500 \mathrm{~nm}$ probes, three $300 \mathrm{~nm}$ probes, two $2 \mu \mathrm{m}$ probes, while only one 1 $\mu \mathrm{m}$ probe and two $100 \mathrm{~nm}$ probe.

\section{Additive micromanufacturing system}

Copper substrates where loaded into the printing chamber and the deposition potential was applied prior to the introduction of the supporting electrolyte to avoid possible copper dissolution. The reservoir of the printing probes was filled with $1 \mu$ of plating solution right before printing. Once the probe was loaded onto the printing head, 500 mbar pressure was applied for $10-15 \mathrm{sec}$ in order to force the plating solution through the cantilever. The full list of files with the printing coordinates was loaded into the system software and the prints were autonomously executed. The entire array of 50 pillars, each $50 \mu \mathrm{m}$ tall, was usually printed in less than $90 \mathrm{~min}$.

\section{Acknowledgements}

This work was supported by the Swiss Innovation Agency Innosuisse (Project Nr. PNFM 18511.1). We thank Joakim Reuteler from the ETH Zurich ScopeM facility for his assistance and support regarding the FIB-SEM. We also acknowledge Cytosurge AG for their support.

Received: ((will be filled in by the editorial staff))

Revised: ((will be filled in by the editorial staff)) Published online: ((will be filled in by the editorial staff))

\section{References}

[1] L. Hirt, A. Reiser, R. Spolenak, T. Zambelli, Adv. Mater. 2017, 29, 17.

[2] P. van Assenbergh, E. Meinders, J. Geraedts, D. Dodou, Small 2018, 14, 20.

[3] J. K. Gansel, M. Thiel, M. S. Rill, M. Decker, K. Bade, V. Saile, G. von Freymann, S. Linden, M. Wegener, Science 2009, 325, 1513.

[4] C. C. J. Alcântara, S. Kim, S. Lee, B. Jang, P. Thakolkaran, J.-Y. Kim, H. Choi, B. J. Nelson, S. Pané, Small 2019, 15, e1805006.

[5] J. Hu, M.-F. Yu, Science 2010, 329, 313. 


\section{WILEY-VCH}

[6] S. Daryadel, A. Behroozfar, S. R. Morsali, S. Moreno, M. Baniasadi, J. Bykova, R. A. Bernal, M. Minary-Jolandan, Nano Lett.2018, 18, 208.

[7] Y.-P. Lin, Y. Zhang, M.-F. Yu, Adv.Mater. Techn. 2019, 4, 1800393

[8] S. K. Seol, D. Kim, S. Lee, J. H. Kim, W. S. Chang, J. T. Kim, Small 2015, 11, 3896.

[9] L. Hirt, S. Ihle, Z. Pan, L. Dorwling-Carter, A. Reiser, J. M. Wheeler, R. Spolenak, J. Vörös, T. Zambelli, Adv. Mater. 2016, 28, 2311.

[10] D. Momotenko, A. Page, M. Adobes-Vidal, P. R. Unwin, ACS Nano 2016, 10, 8871.

[11] A. Meister, M. Gabi, P. Behr, P. Studer, J. Vörös, P. Niedermann, J. Bitterli, J. Polesel-Maris, M. Liley, H. Heinzelmann, T. Zambelli, Nano Lett. 2009, 9, 2501.

[12] A. P. Suryavanshi, M.-F. Yu, Nanotechnology 2007, 18, 105305.

[13] M. Chen, Z. Xu, J. H. Kim, S. K. Seol, J. T. Kim, ACS Nano 2018, 12, 4172.

[14] S. Deladi, N. R. Tas, J. W. Berenschot, G. J. M. Krijnen, M. J. de Boer, J. H. de Boer, M. Péter, M. C. Elwenspoek, Appl. Phys. Lett. 2004, 85, 5361.

[15] E. J. W. Berenschot, N. Burouni, B. Schurink, J. W. van Honschoten, R. G. P. Sanders, R. Truckenmuller, H. V. Jansen, M. C. Elwenspoek, A. A. van Apeldoorn, N. R. Tas, Small 2012, 8, 3823.

[16] L. Dorwling-Carter, M. Aramesh, C. Forró, R. F. Tiefenauer, I. Shorubalko, J. Vörös, T. Zambelli, J. Appl. Phys. 2018, 124, 174902.

[17] M. J. Aebersold, H. Dermutz, L. Demkó, J. F. S. Cogollo, S.-C. Lin, C. Burchert, M. Schneider, D. Ling, C. Forró, H. Han, T. Zambelli, J. Vörös, Chemphyschem 2018, 19, 1234. 


\section{WILEY-VCH}

\section{Table of Contents}

Hollow AFM cantilevers were used as nozzle for electrochemical metal printing. By understanding the effect of applied pressure and aperture size on the lateral voxel size, objects with different voxel sizes were manufactured by changing the applied pressure during the layer-by-layer printing process.

Keyword: electrochemical additive manufacturing

Authors: G. Ercolano*, T. Zambelli*, C. van Nisselroy, J. Vörös, T. Merle, W.W. Koelmans

Title: Multiscale Additive Manufacturing of Metal Microstructures

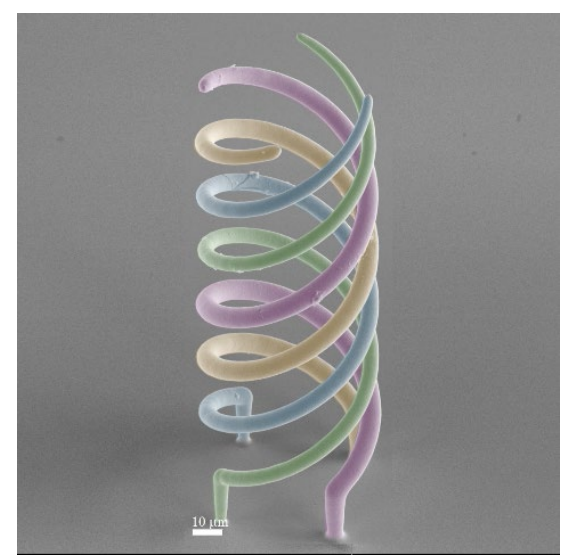




\section{WILEY-VCH}

Copyright WILEY-VCH Verlag GmbH \& Co. KGaA, 69469 Weinheim, Germany, 2018.

\section{Supporting Information}

\section{Multiscale Additive Manufacturing of Metal Microstructures}

Giorgio Ercolano*, Tomaso Zambelli*, Cathelijn van Nisselroy, János Vörös, Thibaut Merle, Wabe W. Koelmans

Dr. Giorgio Ercolano, Thibaut Merle, Dr. Wabe W. Koelmans Exaddon AG, Sägereistrasse 25, 8152, Glattbrugg, Switzerland E-mail: giorgio.ercolano@exaddon.com

Prof. Tomaso Zambelli, Cathelijn van Nisselroy, Prof. János Vörös, Laboratory of Biosensors and Bioelectronics, ETH Zürich, Gloriastrasse 35, 8092, Zurich, Switzerland

E-mail: ztomaso@ethz.ch

\section{Fig. 1S Electrochemical cell}

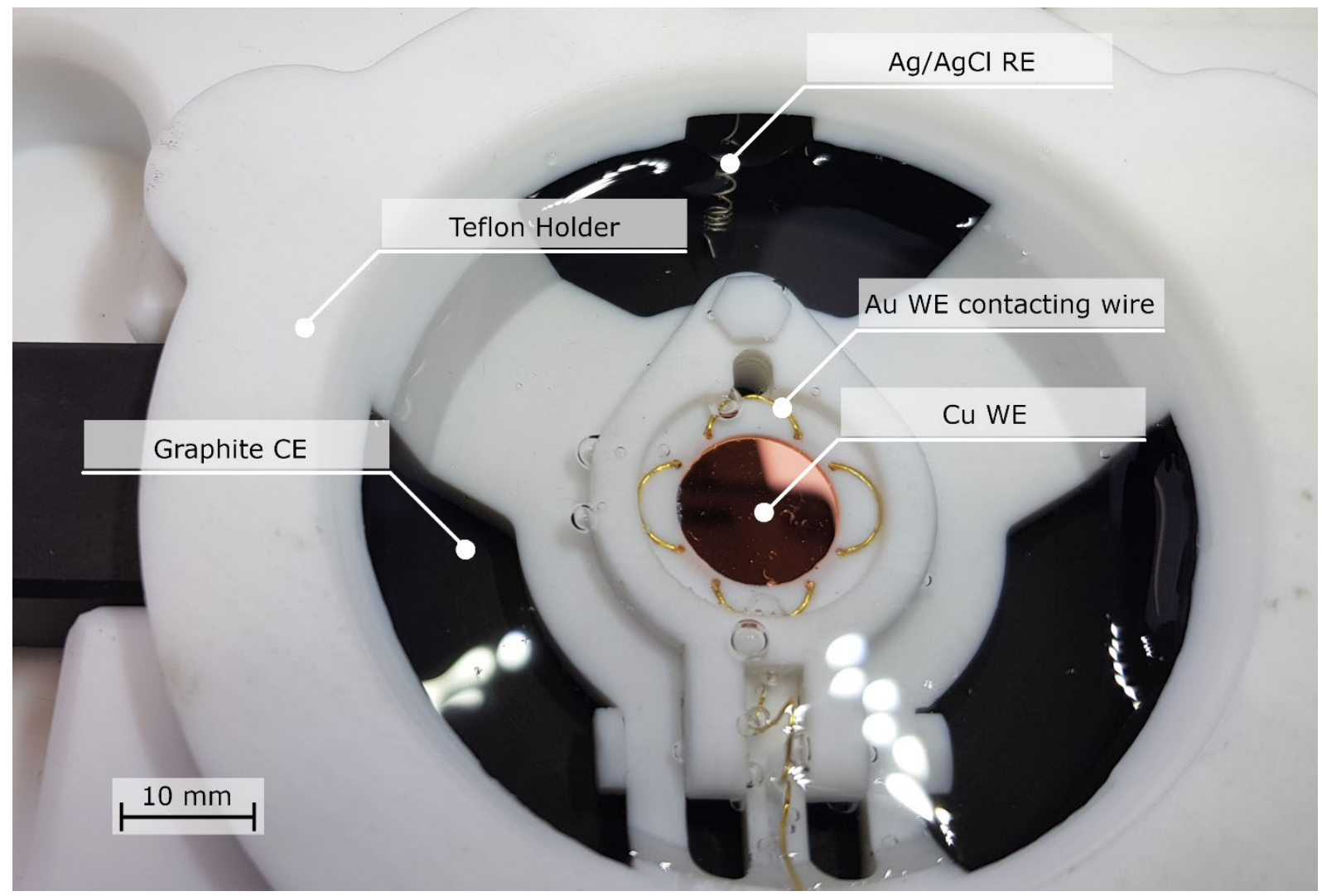

Figure 7S Picture of the printing chamber. 


\section{WILEY-VCH}

Fig. 2S Matlab environment

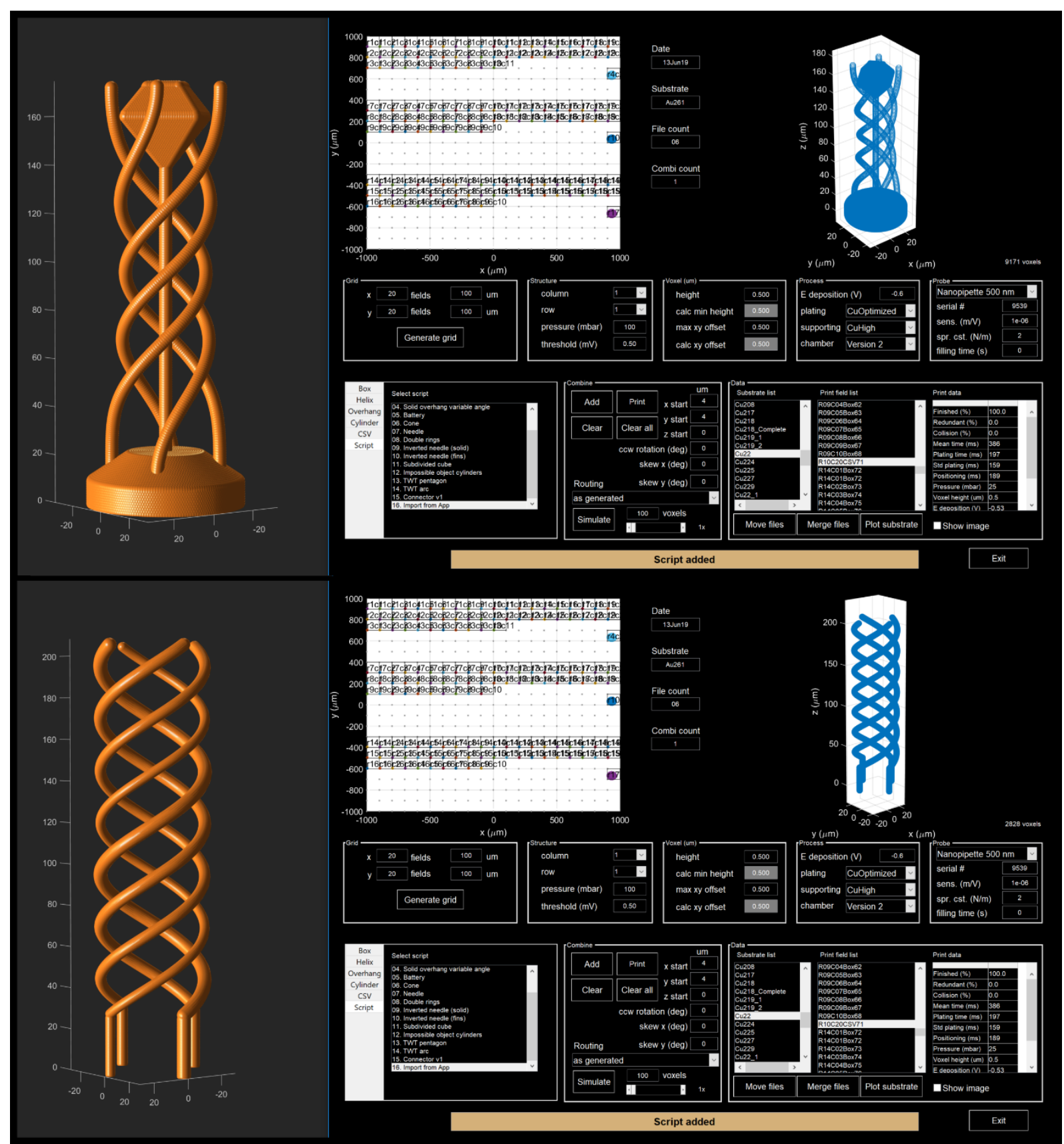

Figure 8S Screenshots of the Matlab environment developed for structure preview, print file generation and archival of prints log, the results of the printing are shown in figure 5 and 6 respectively. 


\section{Derivation of the used equations}

In order to explain the relations between applied pressure $(\Delta \mathrm{P})$ and volumetric deposition speed $(V)$ the system is approximated to two hydrodynamic resistances in series of which only one $\left(R_{t}\right)$ is dependent on the equivalent diameter of the tip aperture $\left(D_{e}\right)$ eq.1.

(1)

$$
\frac{\dot{Q}}{\Delta P}=\frac{1}{\left(R_{c}+R_{t}\left(D_{e}\right)\right)}
$$

The flow $(\dot{Q})$ is related to the volumetric deposition speed by the plating efficiency $(\eta)$.

$$
\dot{Q}=\frac{\dot{V} \rho_{C u}}{\eta c_{C u} M_{C u}}
$$

Substituting eq. 2 into eq. 1 a direct relation between pressure, volumetric deposition speed and, the hydrodynamic resistance is found.

$$
\frac{\dot{V}}{\Delta P}=\frac{\eta c_{C u} M_{C u}}{\rho_{C u}\left(R_{c}+R_{t}\left(D_{e}\right)\right)}
$$

The hydrodynamic resistance can be expressed using the Hagen-Poiseuille solution of the Navier-Stokes equations for the laminar flow of incompressible Newtonian fluids through uniform ducts.

$$
\dot{Q}=\frac{\pi \Delta P_{t}}{128 \mu L_{t}} D_{e}^{4}
$$

The Hagen-Poiseuille can be rewritten in the form of a hydrodynamic resistance function of the equivalent diameter $(\mathrm{De})$ and duct length $\left(\mathrm{L}_{\mathrm{t}}\right)$ of the tip aperture.

$$
\text { (5) } \quad R_{t}\left(D_{e}\right)=\frac{\Delta P_{t}}{\dot{Q}}=\frac{128 \mu L_{t}}{\pi} \frac{1}{D_{e}^{4}}
$$

A final expression for the volumetric deposition speed relation to the applied pressure and tip aperture is found substituting eq.5 into eq. 3

$$
\frac{\dot{V}}{\Delta P}=\frac{\eta c_{C u} M_{C u}}{\rho_{C u}\left(R_{c}+\frac{128 \mu L_{t}}{\pi} \frac{1}{D_{e}^{4}}\right)}
$$




\section{WILEY-VCH}

Finally, assuming that plating efficiency $(\eta), \mathrm{Cu}$ concentration $\left(\mathrm{c}_{\mathrm{Cu}}\right)$, viscosity $(\mu)$ and, aperture duct length $\left(\mathrm{L}_{\mathrm{t}}\right)$ are constant eq. 6 can rewritten as:

(7)

$$
\frac{\dot{V}}{\Delta P}=\frac{1}{C_{1}+\frac{C_{2}}{D_{e}^{4}}}
$$

The expression for the equivalent diameter $D_{e}$ in eqs. 4 to 7 varies with the shape of the duct, in this work the expressions of the equivalent diameter of a rectangular duct (eq. 8a Owen, Trans. Am. Soc. Civ. Eng., 1157-1175 1954) and elliptical duct (eq. 8b Lamb, Hydrodynamics, 6th ed. Dover, New York, 1945) are used for the fibbed and the lithographically obtained apertures respectively.

$$
\text { (8a) } D_{e}=\left(\frac{128 a b^{3}}{\pi k}\right)^{\frac{1}{4}} \quad \text { (8b) } \quad D_{e}=\left(\frac{32 a^{3} b^{3}}{a^{2}+b^{2}}\right)^{\frac{1}{4}}
$$

Symbol Glossary:

Q́: volumetric flow

P: pressure

$\mathrm{R}_{i}$ : hydrodynamic resistance

$\mathrm{D}_{\mathrm{e}}$ : equivalent diameter

Lt: duct length

V́: volumetric deposition speed

$\rho_{\mathrm{Cu}}$ : copper density

$\mathrm{c}_{\mathrm{Cu}}$ : copper $\left(\mathrm{Cu}^{2+}\right)$ concentration

$\mathrm{M}_{\mathrm{Cu}}$ : copper atomic mass

$\eta$ : plating efficiency 